\title{
Interval observer design and control of uncertain non-homogeneous heat equations
}

\author{
Tatiana Kharkovskaia ${ }^{\mathrm{b}}$, Denis Efimov ${ }^{\mathrm{a}}$, Emilia Fridman ${ }^{\mathrm{c}}$, Andrey Polyakov ${ }^{\mathrm{a}}$, \\ Jean-Pierre Richard ${ }^{\text {a }}$ \\ ${ }^{\mathrm{a}}$ Inria, Univ. Lille, Centrale Lille, CNRS, UMR 9189 - CRIStAL, F-59000 Lille, France \\ ${ }^{\mathrm{b}}$ ITMO University, 49 av. Kronverkskiy, 197101 Saint Petersburg, Russia \\ ${ }^{\mathrm{c}}$ School of Electrical Engineering, Tel-Aviv University, Tel-Aviv 69978, Israel
}

\begin{abstract}
The problems of state estimation and observer-based control for heat non-homogeneous equations under distributed in space point measurements are considered. First, an interval observer is designed in the form of Partial Differential Equations (PDEs), without Galerkin projection. Second, conditions of boundedness of the interval observer solutions with non-zero boundary conditions and measurement noise are proposed. Third, the obtained interval estimates are used to design a dynamic outputfeedback stabilizing controller. The advantages of the PDE-based interval observer over the approximation-based one are clearly shown in the numerical example.
\end{abstract}

\section{Introduction}

Due to various technical (complexity of implementation) or economic (price of solution) issues, an explicit measurement of state vector of a dynamical system may be impossible. This is especially the case, for example, in distributed parameter systems, where the system state is a function of the space and time, and only pointwise and discrete measurements are realizable by conventional sensors. Consequently, the system state in these cases has to be reconstructed using estimation algorithms $[1,2,3]$. The most popular approaches in this domain include Luenberger observer and Kalman filter for deterministic and stochastic settings, respectively, which are developed for linear time-invariant models, that is the case where the existing theory disposes plenty of solutions. For nonlinear dynamical systems, the state estimation algorithms are often based on a partial similarity of the plant models to linear ones, or representations in various canonical forms are widely used. The same observations are also valid for control synthesis.

Many physical phenomena can be formalized in terms of PDEs (e.g. sound, heat, electrostatics, electrodynamics, fluid flow, elasticity, or quantum mechanics), whose distributed nature introduces additional level of complexity in design. That is why control and estimation of PDEs are very popular directions of research nowadays $[4,5]$. Frequently, for design of a state estimator or con- trol, the finite-dimensional approximation approach is used $[6,7,8,9]$, then the control or estimation problems are addressed in the framework of finite-dimensional systems using well-known tools. Analysis and design in the original distributed coordinates are more complicated, but also attract attention of many researchers $[5,10,11,12,13,14,15,16]$. In [17] a stabilizing control design with a proportional-discontinuous feedback is proposed for a parabolic PDE with pointwise collocated sensing and actuation, and with in-domain distributed disturbances. The work [18] presents a Luenberger-type observer-based distributed control with non-collocated sensors and actuators.

Inline with the model complexity, the system uncertainty represents another difficulty for synthesis of an estimator or a controller. The uncertainty may consist in unknown parameters or/and external disturbances. Appearance of uncertainty may block a design of a conventional estimator, converging to the ideal value of the state. In this case an interval estimation becomes more attainable: an observer can be constructed such that using input-output information it evaluates the set of admissible values (interval) for the state at each instant of time. The interval width is proportional to the size of the model uncertainty (it has to be minimized by tuning the observer parameters). There are several approaches to design interval/set-membership estimators $[19,20,21]$. This work is devoted to interval observers, 
which form a subclass of set-membership estimators and whose design is based on the theory of monotone systems $[21,22,23,24,25]$. The idea of interval observer construction has been proposed rather recently in [26], but it has already received numerous extensions for various classes of dynamical models. Interval observers for systems described by PDEs have been proposed in [27, 28, 29, 31]. The finite-dimensional approximation approach was applied in [29] using the discretization error estimates from [30], and in [31] for temperature estimation in fuel cells. In [27] the sensitivity function interval estimates are used in order to design an interval observer.

The main contributions of the present paper are as follows. First, an interval observer described by PDEs without applying finite-element approximations is proposed for uncertain distributed parameter systems. Second, an additional design of an output stabilizing control is performed based on interval observations. The estimation error dynamics (also distributed) of the proposed interval observer is guaranteed to be positive following the conditions of positivity of solutions of parabolic PDEs presented in [32]. The stability analysis from [12] is also extended to the considered scenario with non-zero measurement noise and boundary conditions, and further applied for a stabilizing control synthesis for an unstable PDE. An advantage of using interval observers, over Luenberger type observers of [12] and approximation-based interval observer of [29], consists in calculation on-line of accurate bounds explicitly on the given distributed trajectories. It is assumed that the control is spatially distributed influencing the system dynamics through shape functions. Such a hypothesis is introduced to respect the implementation feasibility of the designed control law, since infinitesimal in space variations of the actuator signal cannot be realized in practice. It is worth to highlight that here such a restriction on shape functions is not related with any early lumping procedure. Some preliminary results on an interval PDE observer have been proposed in [33].

The outline of this paper is as follows. After preliminaries in Section 2, and introduction of distributed parameter system properties in Section 3, the interval observer design is given in Section 4. The design of an output control method based on proposed interval observer is considered in Section 5. The results of numerical experiments and a comparison with $[12,29]$ for an unstable parabolic equation are presented in Section 6 .

\section{Preliminaries}

The real numbers are denoted by $\mathbb{R}, \mathbb{R}_{+}=\{\tau \in \mathbb{R}: \tau \geq$ $0\}$. Euclidean norm for a vector $x \in \mathbb{R}^{n}$ will be denoted as $|x|$.

If $X$ is a normed space with the norm $\|\cdot\|_{X}, \Omega \subset \mathbb{R}^{n}$ for some $n \geq 1$ and $\phi: \Omega \rightarrow X$, define

$$
\begin{aligned}
\|\phi\|_{L^{2}(\Omega, X)}^{2} & =\int_{\Omega}\|\phi(s)\|_{X}^{2} d s, \\
\|\phi\|_{L^{\infty}(\Omega, X)} & =\operatorname{ess} \sup _{s \in \Omega}\|\phi(s)\|_{X} .
\end{aligned}
$$

By $L^{\infty}(\Omega, X)$ and $L^{2}(\Omega, X)$ denote the spaces of functions $\Omega \rightarrow X$ with the properties $\|\cdot\|_{L^{\infty}(\Omega, X)}<+\infty$ and $\|\cdot\|_{L^{2}(\Omega, X)}<+\infty$, respectively. Denote $I=[0, \ell]$ for some $\ell>0$, let $C^{k}(I, X)$ be the set of functions having continuous derivatives at least up to order $k \geq 0$ on $I$. For any $q>0$ and an interval $I^{\prime} \subseteq I$ define $W^{q, \infty}\left(I^{\prime}, \mathbb{R}\right)$ as a subset of functions $y \in C^{q-1}\left(I^{\prime}, \mathbb{R}\right)$ with an absolutely continuous $y^{(q-1)}$ and essentially bounded $y^{(q)}$ on $I^{\prime},\|y\|_{W^{q, \infty}}=\sum_{i=0}^{q}\left\|y^{(i)}\right\|_{L^{\infty}\left(I^{\prime}, \mathbb{R}\right)}$. Denote by $H^{q}(I, \mathbb{R})$ with $q \geq 0$ the Sobolev space of functions with derivatives through order $q$ in $L^{2}(I, \mathbb{R})$.

For two functions $\phi_{1}, \phi_{2}: I \rightarrow \mathbb{R}$ their relation $\phi_{1} \leq \phi_{2}$ has to be understood as $\phi_{1}(x) \leq \phi_{2}(x)$ for almost all $x \in I$, the inner product is defined in a standard way:

$$
\left(\phi_{1}, \phi_{2}\right)=\int_{0}^{\ell} \phi_{1}(x) \phi_{2}(x) d x .
$$

For $\phi \in \mathbb{R}$ define two operators $\phi^{+}$and $\phi^{-}$as follows:

$$
\phi^{+}=\max \{0, \phi\}, \phi^{-}=\phi^{+}-\phi .
$$

Lemma 1 [29] Let $s, \underline{s}, \bar{s}: I \rightarrow \mathbb{R}$ admit the relations $\underline{s} \leq s \leq \bar{s}$, then for any $\phi: I \rightarrow \mathbb{R}$ we have

$$
\left(\underline{s}, \phi^{+}\right)-\left(\bar{s}, \phi^{-}\right) \leq(s, \phi) \leq\left(\bar{s}, \phi^{+}\right)-\left(\underline{s}, \phi^{-}\right) .
$$

For later use, we need the following inequalities:

Lemma 2 [34] Wirtinger's Inequality. Let $z \in H^{1}(I, \mathbb{R})$, then

$$
\int_{0}^{\ell} z^{2}(\xi) d \xi \leq \frac{b \ell^{2}}{\pi^{2}} \int_{0}^{\ell}\left[\frac{d z(\xi)}{d \xi}\right]^{2} d \xi,
$$

and if $z(0)=z(\ell)=0$, then $b=1$; if only $z(0)=0$ or $z(\ell)=0$, then $b=4$.

Lemma 3 [35] Poincare's Inequality. Let $z \in H^{1}(I, \mathbb{R})$ with $\int_{0}^{\ell} z(\xi) d \xi=0$, then

$$
\int_{0}^{\ell} z^{2}(\xi) d \xi \leq \frac{\ell^{2}}{\pi^{2}} \int_{0}^{\ell}\left[\frac{d z(\xi)}{d \xi}\right]^{2} d \xi .
$$

\section{Input-to-state stability and positivity of non- homogeneous heat equation}

In this section the basic facts on heat equation and positivity of its solutions are given. 


\subsection{Heat equation}

Consider the following PDE with associated boundary conditions:

$$
\begin{gathered}
\frac{\partial z(x, t)}{\partial t}=L[x, z(x, t)]+r(x, t)+\sum_{j=0}^{p} b_{j}(x) u_{j}(t) \\
\forall(x, t) \in I \times \mathcal{T}, \\
z\left(x, t_{0}\right)=z_{0}(x) \quad \forall x \in I, \\
z(0, t)=\alpha(t), z(\ell, t)=\beta(t) \quad \forall t \in \mathcal{T},
\end{gathered}
$$

where $I=[0, \ell]$ with $0<\ell<+\infty, \mathcal{T}=\left[t_{0}, t_{0}+T\right)$ for $t_{0} \in \mathbb{R}$ and $T>0$,

$$
L(x, z)=\frac{\partial}{\partial x}\left(a(x) \frac{\partial z}{\partial x}\right)+q(x) z
$$

$a \in C^{1}(I, \mathbb{R}), q \in C(I, \mathbb{R})$ and there exist $a_{\min }, a_{\max } \in$ $\mathbb{R}_{+}$such that

$$
0<a_{\min } \leq a(x) \leq a_{\max } \quad \forall x \in I
$$

the boundary conditions $\alpha, \beta \in C^{2}(\mathcal{T}, \mathbb{R})$ and the external input $r \in C^{1}(I \times \mathcal{T}, \mathbb{R})$; the initial conditions $z_{0} \in$ $\mathcal{Z}_{0}=\left\{z_{0} \in H^{2}(I, \mathbb{R}): z_{0}(0)=\alpha(0), z_{0}(\ell)=\beta(0)\right\}$; the controls $u_{j}: \mathcal{T} \rightarrow \mathbb{R}$ are Lipschitz continuous functions. The space domain $I$ is divided into $p+1$ subdomains $I_{j}$ for $j=0,1, \ldots, p$, where the control signals $u_{j}(t)$ are applied through the shape functions $b_{j} \in L^{2}(I,[0,1])$ such that

$$
\begin{cases}b_{j}(x)=0 & x \notin I_{j}, \\ b_{j}(x)=1 & x \in I_{j} .\end{cases}
$$

The controls $u_{j}$ are designed in Section 5 , in Sections 3 and 4 they are assumed to be given and $u_{j} \in L^{\infty}(\mathcal{T}, \mathbb{R})$ for all $j=0,1, \ldots, p$.

\section{Proposition 4 Assume}

$$
a_{\min } \frac{\pi^{2}}{\ell^{2}}>q_{\max }
$$

where $q_{\max }=\sup _{x \in I} q(x)$, then for the solutions of $(3)$ the following estimate is satisfied for all $t \in \mathcal{T}$ :

$$
\begin{aligned}
& \frac{1}{2} \int_{0}^{\ell} z^{2}(x, t) d x \leq e^{-\chi\left(t-t_{0}\right)} \int_{0}^{\ell} w_{0}^{2}(x) d x \\
& +\chi^{-2} \int_{0}^{\ell} \tilde{r}^{2}(x, t) d x+\frac{\ell}{2}\left[\alpha^{2}(t)+\beta^{2}(t)\right],
\end{aligned}
$$

where $\chi=a_{\min } \frac{\pi^{2}}{\ell^{2}}-q_{\max }, w_{0}(x)=z_{0}(x)-\delta\left(x, t_{0}\right)$,

$$
\begin{aligned}
\delta(x, t)= & \alpha(t)+\frac{x}{\ell}(\beta(t)-\alpha(t)), \text { and } \\
\tilde{r}(x, t)= & r(x, t)+\frac{1}{\ell} \frac{\partial a(x)}{\partial x}(\beta(t)-\alpha(t)) \\
& +q(x) \delta(x, t)-\delta_{t}(x, t)+\sum_{j=0}^{p} b_{j}(x) u_{j}(t) .
\end{aligned}
$$

PROOF. Denote $w(x, t)=z(x, t)-\delta(x, t)$, then

$$
\begin{gathered}
\frac{\partial w(x, t)}{\partial t}=L[x, w(x, t)]+\tilde{r}(x, t) \quad \forall(x, t) \in I \times \mathcal{T} \\
w\left(x, t_{0}\right)=w_{0}(x) \quad \forall x \in I \\
w(0, t)=w(\ell, t)=0 \quad \forall t \in \mathcal{T} .
\end{gathered}
$$

We start with the well-posedness analysis of the system (8) under Dirichlet boundary conditions (9). The boundary-value problem (8) can be represented as an abstract differential equation

$$
\dot{\zeta}(t)=A \zeta(t)+F(t, \zeta(t)), t \geq t_{0}, \zeta\left(t_{0}\right)=\zeta_{0}
$$

in the Hilbert space $L^{2}(I, \mathbb{R})$, where the operator $A=\frac{\partial}{\partial x}\left(a(x) \frac{\partial}{\partial x}\right)$ has the dense domain $\mathscr{D}(A)=\{\zeta \in$ $\left.H^{2}(I, \mathbb{R}): \quad \zeta(0)=\zeta(\ell)=0\right\}$. The nonlinear term $F: \mathcal{T} \times L^{2}(I, \mathbb{R}) \rightarrow L^{2}(I, \mathbb{R})$ is defined on functions $\zeta(\cdot, t)$ according to

$$
F(t, \zeta(x, t))=q(x) \zeta(x, t)+\tilde{r}(x, t),
$$

where $\tilde{r}(x, t)$ is given in the equation (7). It is a wellknown fact that $A$ generates a strongly continuous exponentially stable semigroup $\Phi$, which satisfies the inequality $\|\Phi(t)\| \leq \kappa e^{-\rho t}$ for all $t \geq 0$ with some constant $\kappa \geq 1$ and decay rate $\rho>0$.

By introduced restrictions on the initial and boundary conditions $\alpha(t), \beta(t)$ and $\delta(x, t)$ in the PDE (8) and if $u_{j}(t)$ is Lipschitz continuous in $t$, then $F(t, \zeta)$ is Lipschitz continuous in both variables:

$$
\left\|F\left(t_{1}, \zeta_{1}\right)-F\left(t_{2}, \zeta_{2}\right)\right\|_{L^{2}(I, \mathbb{R})} \leq L_{1}\left|t_{1}-t_{2}\right|+L_{2}\left\|\zeta_{1}-\zeta_{2}\right\|_{L^{2}(I, \mathbb{R})}
$$

for all $t_{1}, t_{2} \in \mathcal{T}$ and $\zeta_{1}, \zeta_{2} \in L^{2}(I, \mathbb{R})$, with some $L_{1}>0$ and $L_{2}>0$. Therefore, for all $\zeta_{0} \in \mathscr{D}(A)$ there exists a strong solution of the initial value problem (10) in $C\left(\mathcal{T}, L^{2}(I, \mathbb{R})\right)$ by $[36$, Theorem 6.1.6].

Now consider for (8) the following Lyapunov function

$$
V(t)=\int_{0}^{\ell} w^{2}(x, t) d x .
$$

We have

$$
\begin{aligned}
\dot{V}(t)= & 2 \int_{0}^{\ell} w(x, t)\left[\frac{\partial}{\partial x}\left(a(x) w_{x}(x, t)\right)\right. \\
& +q(x) w(x, t)+\tilde{r}(x, t)] d x .
\end{aligned}
$$


Integrating by parts and substituting the boundary conditions of $w(x, t)$ lead to

$$
\begin{aligned}
\dot{V}(t)= & \left.2 a(x) w(x, t) w_{x}(x, t)\right|_{0} ^{\ell}-2 \int_{0}^{\ell} a(x) w_{x}^{2}(x, t) d x \\
& +2 \int_{0}^{\ell} q(x) w^{2}(x, t)+w(x, t) \tilde{r}(x, t) d x \\
= & 2 \int_{0}^{\ell} q(x) w^{2}(x, t)-a(x) w_{x}^{2}(x, t)+w(x, t) \tilde{r}(x, t) d x .
\end{aligned}
$$

Using Wirtinger's inequality (1) and Young's inequality [34],

$$
2 w(x, t) \tilde{r}(x, t) \leq \chi w^{2}(x, t)+\chi^{-1} \tilde{r}^{2}(x, t),
$$

we obtain (recall that $\chi=a_{\min } \frac{\pi^{2}}{\ell^{2}}-q_{\max }$, see the formulation of the proposition):

$$
\begin{aligned}
\dot{V}(t) \leq & -2\left(a_{\min } \frac{\pi^{2}}{\ell^{2}}-q_{\max }\right) \int_{0}^{\ell} w^{2}(x, t) d x \\
& +2 \int_{0}^{\ell} w(x, t) \tilde{r}(x, t) d x \\
\leq & -\chi V(t)+\chi^{-1} \int_{0}^{\ell} \tilde{r}^{2}(x, t) d x .
\end{aligned}
$$

Therefore, if $\chi>0$ then the system (8) has bounded solutions:

$$
\begin{aligned}
\int_{0}^{\ell} z^{2}(x, t) d x \leq & 2 V(t)+2 \int_{0}^{\ell} \delta^{2}(x, t) d x \\
\leq & 2\left(e^{-\chi\left(t-t_{0}\right)} V\left(t_{0}\right)+\chi^{-2} \int_{0}^{\ell} \tilde{r}^{2}(x, t) d x\right. \\
& \left.+\frac{\ell}{2}\left[\alpha^{2}(t)+\beta^{2}(t)\right]\right)
\end{aligned}
$$

for all $t \in \mathcal{T}$, that completes the proof.

Consequently, Proposition 4 fixes the conditions under which the distributed parameter system (3) possesses the input-to-state stability (ISS) property [37, 38] with respect to the boundary conditions $\alpha, \beta$, the external disturbance $r$ and the control signals $u_{j}$. The main restriction of that proposition is (5) and can be easily validated for a sufficiently small $\ell$.

Note that after a straightforward calculus the estimate from Proposition 4 can be rewritten as follows for all $t \in \mathcal{T}:$

$$
\begin{aligned}
\|z(\cdot, t)\|_{L^{2}(I, \mathbb{R})}^{2} \leq & 4 e^{-\chi\left(t-t_{0}\right)}\left[\left\|z_{0}\right\|_{L^{2}(I, \mathbb{R})}^{2}+\varrho\left(t_{0}\right)\right] \\
& +8 \chi^{-2}\|r(\cdot, t)\|_{L^{2}(I, \mathbb{R})}^{2}+\gamma(t)
\end{aligned}
$$

where $\varrho(t)=\frac{\ell}{2}\left[\alpha^{2}(t)+\beta^{2}(t)\right]$ (weighted norm of the boundary conditions), $\gamma(t)=8 \chi^{-2} \varrho^{\prime}(t)+2\left(1+4 \frac{q_{\max }^{2}}{\chi^{2}}+\right.$ $\left.16 \frac{\partial a_{\max }^{2}}{\chi^{2} \ell^{2}}\right) \varrho(t)$ and $\varrho^{\prime}(t)=\frac{\ell}{2}\left[\dot{\alpha}^{2}(t)+\dot{\beta}^{2}(t)\right]$ (weighted norm of derivative of the boundary conditions) are all bounded functions of time $t \in \mathcal{T}, \partial a_{\max }=\sup _{x \in I} \frac{\partial a(x)}{\partial x}$.

\subsection{Positivity of solutions}

In general, the solution $z(\cdot, t)$ of $(3)$ takes its values in $\mathbb{R}$ and it can change sign with $(x, t) \in I \times \mathcal{T}$. For brevity of presentation of the results of this subsection we will always assume that $u_{j}(t)=0$ for all $t \in \mathcal{T}$ and $j=$ $0,1, \ldots, p$.

Definition 5 The system (3) with $u_{j}(t)=0$ for all $j=$ $0,1, \ldots, p$ is called nonnegative (positive) on the interval $\mathcal{T}$ if for

$$
\alpha(t) \geq 0,, \beta(t) \geq 0, r(x, t) \geq 0 \quad \forall(x, t) \in I \times \mathcal{T}
$$

the implication $z_{0}(x) \geq 0 \Rightarrow z(x, t) \geq 0\left(z_{0}(x)>0 \Rightarrow\right.$ $z(x, t)>0)$ holds for all $(x, t) \in I \times \mathcal{T}$ and for all $z_{0} \in \mathcal{Z}_{0}$.

A well-known example of a nonnegative system is non-homogeneous heat equation defined over $x \in$ $(-\infty,+\infty)$

$$
\begin{gathered}
\frac{\partial \zeta(x, t)}{\partial t}=a \frac{\partial^{2} \zeta(x, t)}{\partial^{2} x}+r(x, t) \quad \forall(x, t) \in \mathbb{R} \times \mathcal{T} \\
\zeta(x, 0)=\zeta_{0}(x) \quad \forall x \in \mathbb{R}
\end{gathered}
$$

where $a>0$ is a constant, $q=0$ and $\zeta_{0}: \mathbb{R} \rightarrow \mathbb{R}_{+}$, whose solution can be calculated analytically using Green's function (fundamental solution or the heat kernel) [39]:

$$
\begin{aligned}
\zeta(x, t)= & \frac{1}{2 \sqrt{\pi a t}} \int_{-\infty}^{+\infty} e^{-\frac{(x-y)^{2}}{4 a t}} \zeta_{0}(y) d y \\
& +\int_{0}^{t} \int_{-\infty}^{+\infty} \frac{e^{-\frac{(x-y)^{2}}{4 a(t-s)}}}{2 \sqrt{\pi a(t-s)}} r(y, s) d y d s .
\end{aligned}
$$

It is straightforward to verify that for nonnegative $\zeta_{0}$ and $r$ the expression in the right-hand side stays nonnegative for all $(x, t) \in \mathbb{R} \times(0,+\infty)$. This conclusion is valid for the case $x \in \mathbb{R}$. However, if $x \in I$, even the homogenous heat equation (11) with $r(x, t)=0$ for all $(x, t) \in I \times \mathcal{T}$, and with the boundary condition

$$
0=\zeta(0, t)=\zeta(\ell, t) \quad \forall t \in \mathcal{T}
$$

admits the solution in the form [39]:

$$
\begin{aligned}
\zeta(x, t) & =\sum_{n=1}^{+\infty} D_{n} \sin \left(\frac{n \pi x}{\ell}\right) e^{-a \frac{n^{2} \pi^{2}}{\ell^{2}} t} \\
D_{n} & =\frac{2}{\ell} \int_{0}^{\ell} \zeta_{0}(x) \sin \left(\frac{n \pi x}{\ell}\right) d x
\end{aligned}
$$


whose positivity is less trivial to establish.

For this reason, using Maximum principle [40] the following general result has been proven in [32]:

Proposition 6 Let $\alpha, \beta \in L^{2}\left(\mathcal{T}, \mathbb{R}_{+}\right), r \in L^{2}(I \times$ $\left.\mathcal{T}, \mathbb{R}_{+}\right)$and $z_{0} \in H^{1}\left(I, \mathbb{R}_{+}\right)$, then

$$
z(x, t) \geq 0 \quad \forall(x, t) \in I \times \mathcal{T},
$$

i.e. (3) with $u_{j}(t)=0$ for all $j=0,1, \ldots, p$ is nonnegative on the interval $\mathcal{T}$.

Therefore, if boundary and initial conditions, and external inputs, take only nonnegative values, then the solutions of (3) possess the same property.

\section{Interval observer design for the heat equation}

Consider (3) with some uncertain boundary conditions $\alpha, \beta \in C^{2}(\mathcal{T}, \mathbb{R})$, an uncertain external input $r \in C(I \times$ $\mathcal{T}, \mathbb{R})$ and initial conditions $z_{0} \in \mathcal{Z}_{0}$, and assume that the state $z(x, t)$ is available for measurements in certain points $0<x_{1}^{m}<x_{2}^{m}<\cdots<x_{p}^{m}<\ell$ :

$$
y_{j}(t)=z\left(x_{j}^{m}, t\right)+\nu_{j}(t), j=1, \ldots, p,
$$

where $y(t)=\left[y_{1}(t), \ldots, y_{p}(t)\right]^{T} \in \mathbb{R}^{p}$ is the measured output signal, $\nu(t)=\left[\nu_{1}(t), \ldots, \nu_{p}(t)\right] \in \mathbb{R}^{p}$ is the output disturbance (measurement noise). Design of a conventional observer under similar conditions has been studied in $[12,13]$. Further, to simplify the technical presentation (to simplify the proof of well-posedness of the estimation error dynamics) we assume differentiability of the output disturbance:

\section{Assumption 1 Let $\nu \in C^{2}\left(\mathcal{T}, \mathbb{R}^{p}\right)$}

A goal of the work consists in design of interval observers for the distributed parameter system (3), (13). For this purpose we need the following hypothesis.

Assumption 2 Let $\underline{z}_{0} \leq z_{0} \leq \bar{z}_{0}$ for some known $\underline{z}_{0}, \bar{z}_{0} \in \mathcal{Z}_{0}$, let also functions $\underline{\alpha}, \bar{\alpha}, \beta, \bar{\beta} \in C^{2}(\mathcal{T}, \mathbb{R})$, $\underline{r}, \bar{r} \in C^{1}(I \times \mathcal{T}, \mathbb{R})$ and a constant $\nu_{0}>0$ be given such that for all $(x, t) \in I \times \mathcal{T}$ :

$$
\begin{gathered}
\underline{\alpha}(t) \leq \alpha(t) \leq \bar{\alpha}(t), \underline{\beta}(t) \leq \beta(t) \leq \bar{\beta}(t), \\
\underline{r}(x, t) \leq r(x, t) \leq \bar{r}(x, t),|\nu(t)| \leq \nu_{0} .
\end{gathered}
$$

Thus, by Assumption 2 five intervals, $[\underline{\alpha}(t), \bar{\alpha}(t)]$, $[\beta(t), \bar{\beta}(t)],\left[\underline{z}_{0}, \bar{z}_{0}\right],[\underline{r}(x, t), \bar{r}(x, t)]$ and $\left[-\nu_{0}, \nu_{0}\right]$, determine for all $(x, t) \in I \times \mathcal{T}$ in (3), (13) the uncertainty of the values for $\alpha(t), \beta(t), z_{0}, r(x, t)$ and $\nu(t)$, respectively.
Remark 7 These imperfections can be related with various reasons, e.g. unknown parameters, external signals, nonlinearities, etc., but they have to be included in the corresponding intervals. For example, consider even more complicated case, let

$$
r(z, x, t)=\theta_{1} \tilde{r}(x, t)+\theta_{2}(z, x, t),
$$

where

$$
\theta_{1} \in\left[\underline{\theta}_{1}, \bar{\theta}_{1}\right]
$$

is an unknown parameter taking values in the given interval $\left[\underline{\theta}_{1}, \bar{\theta}_{1}\right], \tilde{r}: I \times \mathcal{T} \rightarrow \mathbb{R}_{+}$is a known function and $\theta_{2}: L^{2}(I, \mathbb{R}) \times I \times \mathcal{T} \rightarrow\left[\underline{\theta}_{2}, \bar{\theta}_{2}\right]$ is an unknown function taking values in the given set $\left[\underline{\theta}_{2}, \bar{\theta}_{2}\right]$. Then

$r(z, x, t) \in\left[\underline{\theta}_{1} \tilde{r}(x, t)+\underline{\theta}_{2}, \bar{\theta}_{1} \tilde{r}(x, t)+\bar{\theta}_{2}\right]=[\underline{r}(x, t), \bar{r}(x, t)]$ and this case also can be studied in the same way as (3).

The simplest interval observer for (3) under the introduced assumptions is as follows for $j=0,1, \ldots, p$ :

$$
\begin{gathered}
\frac{\partial \bar{z}(x, t)}{\partial t}=L[x, \bar{z}(x, t)]+\bar{r}(x, t)+b_{j}(x) u_{j}(t) \\
\forall(x, t) \in I_{j} \times \mathcal{T}, \\
\bar{z}\left(x, t_{0}\right)=\bar{z}_{0}(x) \quad \forall x \in I_{j}, \\
\bar{z}\left(x_{j}^{m}, t\right)=\bar{Z}_{j}(t), \bar{z}\left(x_{j+1}^{m}, t\right)=\bar{Z}_{j+1}(t) \quad \forall t \in \mathcal{T} ; \quad \\
\frac{\partial \underline{z}(x, t)}{\partial t}=L[x, \underline{z}(x, t)]+\underline{r}(x, t)+b_{j}(x) u_{j}(t) \\
\forall(x, t) \in I_{j} \times \mathcal{T}, \\
\underline{z}^{\prime}\left(x, t_{0}\right)=\underline{z}_{0}(x) \quad \forall x \in I_{j}, \\
\underline{z}\left(x_{j}^{m}, t\right)=\underline{Z}_{j}(t), \underline{z}\left(x_{j+1}^{m}, t\right)=\underline{Z}_{j+1}(t) \quad \forall t \in \mathcal{T},
\end{gathered}
$$

where $\bar{z} \in C\left(\mathcal{T}, L^{2}(I, \mathbb{R})\right)$ and $\underline{z} \in C\left(\mathcal{T}, L^{2}(I, \mathbb{R})\right)$ are upper and lower estimates of the solution $z(x, t) ; I_{j}=$ $\left[x_{j}^{m}, x_{j+1}^{m}\right]$ with $x_{0}^{m}=0$ and $x_{p+1}^{m}=\ell$; the upper and lower estimates for the boundary conditions are

$$
\begin{gathered}
\bar{Z}(t)=\left[\bar{Z}_{0}(t), \ldots, \bar{Z}_{p}(t)\right]^{T} \\
=\left[\bar{\alpha}(t), y_{1}(t)+\nu_{0}, \ldots, y_{p}(t)+\nu_{0}, \bar{\beta}(t)\right]^{T}, \\
\underline{Z}(t)=\left[\underline{Z}_{0}(t), \ldots, \underline{Z}_{p}(t)\right]^{T} \\
=\left[\underline{\alpha}(t), y_{1}(t)-\nu_{0}, \ldots, y_{p}(t)-\nu_{0}, \underline{\beta}(t)\right]^{T} .
\end{gathered}
$$

Therefore, the domain $I$ of the solution of (3) is divided on $p+1$ subdomains with appropriate boundary conditions. It is related with the manner the output injection is applied. In (14) the use of the output injection directly in the observer right-hand side is avoided since the analysis of positivity of the estimation error dynamics, which is obligatory for an interval observer and given below, is straightforward if the output injection is present at the boundaries, but it is more evolved in other cases. 
The upper and the lower interval estimation errors for (3) and (14) can be introduced as follows:

$$
\bar{e}(x, t)=\bar{z}(x, t)-z(x, t), \underline{e}(x, t)=z(x, t)-\underline{z}(x, t),
$$

whose dynamics take the form for $j=0,1, \ldots, p$ :

$$
\begin{aligned}
\frac{\partial \bar{e}(x, t)}{\partial t}= & L[x, \bar{e}(x, t)]+\bar{r}(x, t) \\
& -r(x, t) \quad \forall(x, t) \in I_{j} \times \mathcal{T}, \\
\bar{e}\left(x, t_{0}\right)= & \bar{z}_{0}(x)-z_{0}(x) \quad \forall x \in I_{j}, \\
\bar{e}\left(x_{j}^{m}, t\right)= & \bar{Z}_{j}(t)-z\left(x_{j}^{m}, t\right) \quad \forall t \in \mathcal{T}, \\
\bar{e}\left(x_{j+1}^{m}, t\right)= & \bar{Z}_{j+1}(t)-z\left(x_{j+1}^{m}, t\right) \quad \forall t \in \mathcal{T} ; \\
\frac{\partial \underline{e}(x, t)}{\partial t}= & L[x, \underline{e}(x, t)]+r(x, t) \\
& -\underline{r}(x, t) \quad \forall(x, t) \in I_{j} \times \mathcal{T}, \\
\underline{e}\left(x, t_{0}\right)= & z_{0}(x)-\underline{z}_{0}(x) \quad \forall x \in I_{j}, \\
\underline{e}\left(x_{j}^{m}, t\right)= & z\left(x_{j}^{m}, t\right)-\underline{Z}_{j}(t) \quad \forall t \in \mathcal{T}, \\
\underline{e}\left(x_{j+1}^{m}, t\right)= & z\left(x_{j+1}^{m}, t\right)-\underline{Z}_{j+1}(t) \quad \forall t \in \mathcal{T} .
\end{aligned}
$$

Theorem 8 Let assumptions 1 and 2 be satisfied, then in (3), (14):

$$
\underline{z}(x, t) \leq z(x, t) \leq \bar{z}(x, t) \quad \forall(x, t) \in I \times \mathcal{T} .
$$

In addition, if

$$
\Delta x^{m}<\pi \sqrt{\frac{a_{\min }}{q_{\max }}}
$$

where $\Delta x^{m}=\max _{j \in\{0,1, \ldots, p\}}\left(x_{j+1}^{m}-x_{j}^{m}\right)$, then for all $t \in \mathcal{T}$ :

$$
\begin{aligned}
\|\bar{z}(\cdot, t)-z(\cdot, t)\|_{L^{2}(I, \mathbb{R}) \leq}^{2} \leq & 4 e^{-\chi\left(t-t_{0}\right)}\left[\left\|\bar{z}_{0}-z_{0}\right\|_{L^{2}(I, \mathbb{R})}^{2}+\bar{\varrho}\left(t_{0}\right)\right] \\
& +8 \chi^{-2}\|\bar{r}(\cdot, t)-r(\cdot, t)\|_{L^{2}(I, \mathbb{R})}^{2}+\bar{\gamma}(t), \\
\|z(\cdot, t)-\underline{z}(\cdot, t)\|_{L^{2}(I, \mathbb{R}) \leq}^{2} \leq & 4 e^{-\chi\left(t-t_{0}\right)}\left[\left\|z_{0}-\underline{z}_{0}\right\|_{L^{2}(I, \mathbb{R})}^{2}+\underline{\varrho}\left(t_{0}\right)\right] \\
& +8 \chi^{-2}\|r(\cdot, t)-\underline{r}(\cdot, t)\|_{L^{2}(I, \mathbb{R})}^{2}+\underline{\gamma}(t),
\end{aligned}
$$

where $\bar{\varrho}(t)=\ell\|\bar{Z}(t)-Z(t)\|^{2}, \bar{\varrho}^{\prime}(t)=\ell\|\dot{\bar{Z}}(t)-\dot{Z}(t)\|^{2}$, $\bar{\gamma}(t)=8 \chi^{-2} \bar{\varrho}^{\prime}(t)+2\left(1+4 \frac{q_{\max }^{2}}{\chi^{2}}+16 \frac{\partial a_{\max }^{2}}{\chi^{2} \ell^{2}}\right) \bar{\varrho}(t)$, $\underline{\varrho}(t)=\ell\|Z(t)-\underline{Z}(t)\|^{2}, \underline{\varrho}(t)=\ell\|\dot{Z}(t)-\underline{Z}(t)\|^{2}$, $\underline{\gamma}(t)=8 \chi^{-2} \underline{\varrho}^{\prime}(t)+2\left(1+4 \frac{q_{\max }^{2}}{\chi^{2}}+16 \frac{\partial a_{\max }^{2}}{\chi^{2} \ell^{2}}\right) \underline{\varrho}(t)$ and

$$
Z(t)=\left[\alpha(t), y^{T}(t)-\nu^{T}(t), \beta(t)\right]^{T} .
$$

PROOF. Under Assumption 2, for all $(x, t) \in I \times \mathcal{T}$, in (16) the external inputs

$$
\bar{r}(x, t)-r(x, t) \geq 0, r(x, t)-\underline{r}(x, t) \geq 0,
$$

the initial conditions

$$
\bar{z}_{0}(x)-z_{0}(x) \geq 0, z_{0}(x)-\underline{z}_{0}(x) \geq 0,
$$

the boundary conditions

$$
\begin{gathered}
\bar{e}\left(x_{0}^{m}, t\right)=\bar{\alpha}(t)-\alpha(t) \geq 0, \\
\bar{e}\left(x_{i}^{m}, t\right)=y_{i}(t)+\nu_{0}-z\left(x_{i}^{m}, t\right) \\
=\nu(t)+\nu_{0} \geq 0, i=1, \ldots, p, \\
\bar{e}\left(x_{p+1}^{m}, t\right)=\bar{\beta}(t)-\beta(t) \geq 0 ; \\
\underline{e}\left(x_{0}^{m}, t\right)=\alpha(t)-\underline{\alpha}(t) \geq 0, \\
\underline{e}\left(x_{i}^{m}, t\right)=z\left(x_{i}^{m}, t\right)-y_{i}(t)+\nu_{0} \\
=\nu_{0}-\nu(t) \geq 0, i=1, \ldots, p, \\
\underline{e}\left(x_{p+1}^{m}, t\right)=\beta(t)-\underline{\beta}(t) \geq 0,
\end{gathered}
$$

are all nonnegative. Therefore, according to Proposition 6 the PDE (16) is nonnegative on the interval $\mathcal{T}$, which implies the required interval estimates by the definition of $\bar{e}$ and $\underline{e}$.

Boundedness of $\bar{z}, \underline{z}$ for all $t \geq t_{0}$ follows from Proposition 4 and the condition (18) under Assumption 1.

Remark 9 Following the idea from [14], the wellposedness of (14) can be established by showing the well-posedness of the estimation errors (15), which satisfy the equations (16). By the introduced constraints on the system parameters, $r(x, t), \underline{r}(x, t)$ and $\bar{r}(x, t)$; initial conditions $z_{0}(x), \underline{z}_{0}(x)$ and $\bar{z}_{0}(x)$, and boundary conditions for the error dynamics (16) (recall (19) for $\alpha, \beta, \underline{\alpha}, \bar{\alpha}, \underline{\beta}, \bar{\beta} \in C^{2}(\mathcal{T}, \mathbb{R})$ and $\nu \in C^{2}\left(\mathbb{R}_{+}, \mathbb{R}^{p}\right)$ by assumptions 1 and 2), and for $\bar{z}_{0}-z_{0}, z_{0}-\underline{z}_{0} \in \mathscr{D}(A)$ there exists a strong solution $\underline{e}, \bar{e} \in C\left(\mathcal{T}, L^{2}(I, \mathbb{R})\right)$ of initial value problem (16) with $\underline{e}(t, \cdot), \bar{e}(t, \cdot) \in \mathscr{D}(A)$ by [36, Corollary 4.2.5]. Therefore, if $\underline{e}(t, \cdot), \bar{e}(t, \cdot) \in \mathscr{D}(A)$ and $z(t, \cdot) \in \mathscr{D}(A)$, then there exists a unique solution $\underline{z}, \bar{z} \in C\left(\mathcal{T}, L^{2}(I, \mathbb{R})\right)$ to the interval observer system (14) with $\underline{z}(t, \cdot), \bar{z}(t, \cdot) \in \mathscr{D}(A)$ for all $t \in \mathcal{T}$.

It is a well-known fact that the system (16) can be unstable if the function $q$ takes sufficiently big values [41]. In [12] it has been proven, for $\alpha(t)=\beta(t)=0$ and $\nu(t)=0$, that the observer (14) is asymptotically stable if the difference $\Delta x^{m}$ is sufficiently small (i.e. there are sufficient quantity of sensors uniformly distributed in $I$ ). The presented Theorem 8 ensures positiveness of the interval estimation errors and boundedness of the interval estimates $\bar{z}$ and $\underline{z}$ in the presence of non-zero boundary conditions $\alpha(t), \beta(t)$ and measurement noise $\nu(t)$.

\section{$5 \quad$ Stabilizing control}

In this section the interval observer (14) is used for design of a control law ensuring stabilization of (3).

The main restriction on stability for the system (3) is $q_{\max }<a_{\min } \frac{\pi^{2}}{\ell^{2}}$. The inequality (18) imposes the same property for the interval observer (14): if the difference $\Delta x^{m}$ is sufficiently small, which means that the quantity of measurement points is sufficiently high, then the 
observer estimation error is bounded, but it does not imply stability of the original system. To overcome this restriction, let us consider together the system (3) and the interval observer (14), designed in the Section 4, both endowed with control input $u_{j}(t) \in H^{1}(\mathcal{T}, \mathbb{R})$ through the shape functions $b_{j}(x) \in L^{2}(I, \mathbb{R})$ on each space subdomain $I_{j}$, where the control is chosen as an interval observer state feedback:

$$
u_{j}(t)=-\frac{K_{j}}{\Delta x_{j}^{m}} \int_{x_{j}^{m}}^{x_{j+1}^{m}}(\underline{z}(\xi, t)+\bar{z}(\xi, t)) d \xi, j=0, \ldots, p,
$$

where $K_{j}$ are the sequential feedback gains to be designed on each $I_{j}, K_{j}>0$ and

$$
\Delta x_{j}^{m}=\left(x_{j+1}^{m}-x_{j}^{m}\right) \quad \forall j \in\{0,1, \ldots, p\} .
$$

Remark 10 For brevity we consider the same number of sensors and actuators with collocated subintervals $I_{j}$. It is not difficult to extend our results to the non-collocated case by modifying arguments of [15]. This is because our design is based on separation of the controller and the observer designs. While the observer part of this paper is completely new, the controller part is based on a modification of the existing controller method from [16]. Our modification of the existing controller design is as follows: we use transformation to move boundary disturbances into the right-hand side of PDE and employ a special structure of the controller based on the interval observer. Then the ISS analysis of the closed-loop system follows the existing method for controller design. Thus, by modifying arguments of Section 2 of [15], it is possible to achieve ISS by using a boundary controller at $x=\ell$ via the backstepping.

Thus, the control is applied in order to ensure boundedness of the observer estimates $\underline{z}(x, t), \bar{z}(x, t)$, that in its turn (since $\underline{z}(x, t) \leq z(x, t) \leq \bar{z}(x, t)$ for all $(x, t) \in I \times \mathcal{T}$, see Theorem 8) will provide boundedness of $z(x, t)$ as in [42]. Recall the shape functions $(4) b_{j}(x)=1$ on $I_{j}$ and $b_{j}(x)=0$ if $x \notin I_{j}$ and substitute the control (20) in (3) on interval $I_{j}$ for all $j=0, \ldots p$ :

$$
\begin{gathered}
\frac{\partial z(x, t)}{\partial t}=\frac{\partial}{\partial x}\left(a(x) z_{x}(x, t)\right)+q(x) z(x, t)+r(x, t) \\
-\frac{K_{j}}{\Delta} x_{j}^{m} \int_{x_{j}^{m}}^{x_{j+1}^{m}}(\underline{z}(\xi, t)+\bar{z}(\xi, t)) d \xi, \quad \forall(x, t) \in I_{j} \times \mathcal{T} .
\end{gathered}
$$

We consider the same shift for the system as before $\delta(x, t)=\alpha(t)+\frac{x}{\ell}(\beta(t)-\alpha(t))$, then the new state variable (as in the proof of Proposition 4) is $w(x, t)=z(x, t)-\delta(x, t)$, and it satisfies the following PDE with zero boundary conditions:

$$
\begin{gathered}
\frac{\partial w(x, t)}{\partial t}=\frac{\partial}{\partial x}\left(a(x) w_{x}(x, t)\right)+q(x) w(x, t)+\tilde{r}(x, t) \\
-\sum_{j=0}^{p} b_{j}(x) \frac{K_{j}}{\Delta} x_{j}^{m} \int_{x_{j}^{m}}^{x_{j+1}^{m}}(\underline{z}(\xi, t)+\bar{z}(\xi, t)) d \xi \quad \forall(x, t) \in I \times \mathcal{T}, \\
w\left(x, t_{0}\right)=w_{0}(x) \quad \forall x \in I, \\
w(0, t)=w(\ell, t)=0 \quad \forall t \in \mathcal{T} .
\end{gathered}
$$

where $\tilde{r}(x, t)=r(x, t)+\frac{1}{\ell} \frac{\partial a(x)}{\partial x}(\beta(t)-\alpha(t))+$ $q(x) \delta(x, t)-\delta_{t}(x, t)$ (before this auxiliary perturbation also included the control part $\left.\sum_{j=0}^{p} b_{j}(x) u_{j}(t)\right)$.

Consider the interval observer error dynamics (16), which is nonnegative by Theorem 8 and bounded if the condition (18) is satisfied. Recall the relations $\underline{z}(x, t)=z(x, t)-\underline{e}(x, t)$ and $\bar{z}(x, t)=z(x, t)+\bar{e}(x, t)$ and substitute them into the dynamics of $w(x, t)$ :

$$
\begin{gathered}
\frac{\partial w(x, t)}{\partial t}=\frac{\partial}{\partial x}\left(a(x) w_{x}(x, t)\right)+q(x) w(x, t)+\tilde{r}^{*}(x, t) \\
+\sum_{j=0}^{p} b_{j}(x) \frac{K_{j}}{\Delta} x_{j}^{m} \int_{x_{j}^{m}}^{x_{j+1}^{m}}(\underline{e}(\xi, t)-\bar{e}(\xi, t)) d \xi \\
-2 \sum_{j=0}^{p} b_{j}(x) \frac{K_{j}}{\Delta} x_{j}^{m} \int_{x_{j}^{m}}^{x_{j+1}^{m}} w(\xi, t) d \xi \quad \forall(x, t) \in I \times \mathcal{T},
\end{gathered}
$$

where $\tilde{r}^{*}(x, t)=\tilde{r}(x, t)-2 \sum_{j=0}^{p} b_{j}(x) \frac{K_{j}}{\Delta} x_{j}^{m} \int_{x_{j}^{m}}^{x_{j+1}^{m}} \delta(\xi, t) d \xi$. Since $\underline{e}(x, t) \geq 0, \bar{e}(x, t) \geq 0$ and bounded under the condition (18), the terms $\int_{x_{j}^{m}}^{x_{j+1}^{m}}(\underline{e}(\xi, t)-\bar{e}(\xi, t)) d \xi$ can be made a part of a new disturbance

$R(x, t)=\tilde{r}^{*}(x, t)+\sum_{j=0}^{p} b_{j}(x) \frac{K_{j}}{\Delta} x_{j}^{m} \int_{x_{j}^{m}}^{x_{j+1}^{m}}(\underline{e}(\xi, t)-\bar{e}(\xi, t)) d \xi$,

then

$$
\begin{aligned}
& \frac{\partial w(x, t)}{\partial t}=\frac{\partial}{\partial x}\left(a(x) w_{x}(x, t)\right)+q(x) w(x, t)+R(x, t) \\
& -2 \sum_{j=0}^{p} b_{j}(x) \frac{K_{j}}{\Delta} x_{j}^{m} \int_{x_{j}^{m}}^{x_{j+1}^{m}} w(\xi, t) d \xi \quad \forall(x, t) \in I \times \mathcal{T} .
\end{aligned}
$$

In order to analyze the influence of the integral feedback, let us use the relation

$$
\frac{1}{\Delta} x_{j}^{m} \int_{x_{j}^{m}}^{x_{j+1}^{m}} w(\xi, t) d \xi=w(x, t)-f(x, t), \quad x \in I_{j},
$$

proposed in [16], where

$$
f(x, t) \triangleq \frac{1}{\Delta} x_{j}^{m} \int_{x_{j}^{m}}^{x_{j+1}^{m}}[w(x, t)-w(\xi, t)] d \xi
$$


is a piecewise continuous function and $\frac{\partial f}{\partial x}=\frac{\partial w}{\partial x}$. Finally, the following closed-loop system has been obtained:

$$
\begin{gathered}
\frac{\partial w(x, t)}{\partial t}=\frac{\partial}{\partial x}\left(a(x) w_{x}(x, t)\right)+q(x) w(x, t)+R(x, t) \\
-2 \sum_{j=0}^{p} K_{j} b_{j}(x) w(x, t)+2 \sum_{j=0}^{p} K_{j} b_{j}(x) f(x, t) .
\end{gathered}
$$

Validity of the interval inclusion (17) can be proven repeating the same arguments as in Theorem 8 since the observer design is independent on the form of control. To analyze stability of the closed-loop system (22) let us consider a Lyapunov function:

$$
V(t)=\int_{0}^{\ell} w^{2}(x, t) d x
$$

whose derivative takes the form for any $\gamma>0$ and $\kappa>0$ :

$$
\begin{gathered}
\dot{V}(t)+2 \kappa V(t)-\gamma^{2} \int_{0}^{\ell} R(x, t)^{2} d x= \\
=2 \int_{0}^{\ell} w(x, t)\left[\frac{\partial}{\partial x}\left(a(x) w_{x}(x, t)\right)\right. \\
+q(x) w(x, t)+R(x, t)] d x+2 \kappa \int_{0}^{\ell} w^{2}(x, t) d x \\
-\gamma^{2} \int_{0}^{\ell} R^{2}(x, t) d x-4 \int_{0}^{\ell}\left[\sum_{j=0}^{p} K_{j} b_{j}(x) w(x, t)\right] w(x, t) d x \\
+4 \int_{0}^{\ell}\left[\sum_{j=0}^{p} K_{j} b_{j}(x) f(x, t)\right] w(x, t) d x .
\end{gathered}
$$

Integration by parts and substitution of the boundary conditions for $w(x, t)$ lead to

$2 \int_{0}^{\ell} w(x, t) \frac{\partial}{\partial x}\left(a(x) w_{x}(x, t)\right) d x \leq-2 a_{\min } \int_{0}^{\ell} w_{x}^{2}(x, t) d x$.

The function $f(x, t)$ has the zero average $\int_{x_{j}^{m}}^{x_{j+1}^{m}} f(x, t) d x=$ 0 and $f_{x}=w_{x}$, and by applying the Poincare's inequality (2) on subdomains $I_{j}$ the following upper estimate is obtained:

$-2 a_{\min } \int_{x_{j}^{m}}^{x_{j+1}^{m}} w_{x}^{2}(x, t) d x \leq-2 a_{\min } \frac{\pi^{2}}{\left(\Delta x_{j}^{m}\right)^{2}} \int_{x_{j}^{m}}^{x_{j+1}^{m}} f^{2}(x, t) d x$,

then

$$
\begin{gathered}
-2 a_{\min } \int_{0}^{\ell} w_{x}^{2}(x, t) d x=-2 a_{\min } \sum_{j=0}^{p} \int_{x_{j}^{m}}^{x_{j+1}^{m}} w_{x}^{2}(x, t) d x \\
\leq-2 a_{\min } \frac{\pi^{2}}{\left(\Delta x_{j}^{m}\right)^{2}} \sum_{j=0}^{p} \int_{x_{j}^{m}}^{x_{j+1}^{m}} f^{2}(x, t) d x .
\end{gathered}
$$

The next term of (23) can be rewritten using the fact that $b_{j}(x)=1$ on $I_{j}$ in (4) and under a mild simplifying restriction that $K_{j}=K$ for all $j=0, \ldots p$ :

$-4 \int_{0}^{\ell}\left[\sum_{j=0}^{p} K_{j} b_{j}(x) w^{2}(x, t)\right] d x=-4 K \sum_{j=0}^{p} \int_{x_{j}^{m}}^{x_{j+1}^{m}} w^{2}(x, t) d x$.

And the cross term of (23) can be treated in the same way:

$$
\begin{aligned}
& 4 \int_{0}^{\ell}\left[\sum_{j=0}^{p} K_{j} b_{j}(x) f(x, t) w(x, t)\right] d x \\
& =4 K \sum_{j=0}^{p} \int_{x_{j}^{m}}^{x_{j+1}^{m}} w(x, t) f(x, t) d x
\end{aligned}
$$

Therefore, using an upper bound $\int_{0}^{\ell} q(x) w^{2}(x, t) \leq$ $q_{\max } \int_{0}^{\ell} w^{2}(x, t)$ and denoting $\eta^{\top}=[w(x, t) f(x, t) R(x, t)]$, we get

$\dot{V}(t)+2 \kappa V(t)-\gamma^{2} \int_{0}^{\ell} R(x, t)^{2} d x \leq \sum_{j=0}^{p} \int_{x_{j}^{m}}^{x_{j+1}^{m}} \eta^{\top} \Phi \eta d x \leq 0$

provided that

$$
\Phi \triangleq\left[\begin{array}{ccc}
2\left(\kappa+q_{\max }-2 K\right) & 2 K & 1 \\
2 K & \frac{-2 a_{\min } \pi^{2}}{\left(\Delta x^{m}\right)^{2}} & 0 \\
1 & 0 & -\gamma^{2}
\end{array}\right] \leq 0
$$

for $\Delta x^{m}=\max _{j \in\{0,1, \ldots, p\}} \Delta x_{j}^{m}$. Using the Schur complement the above inequality is satisfied if $\left[\begin{array}{cc}\frac{2 a_{\min } \pi^{2}}{\Delta x^{m}} & 0 \\ 0 & \gamma^{2}\end{array}\right]>0,2 K-\frac{\left(\Delta x^{m}\right)^{2}}{a_{\min } \pi^{2}} K^{2}-\kappa-q_{\max }-\frac{1}{2} \gamma^{-2} \geq 0$,

where the first property is valid by proposed construction and the last one is a quadratic inequality with respect to $K$. Using the imposed restriction (18) there exists $\varrho>0$ such that

$$
\frac{\left(\Delta x^{m}\right)^{2}}{a_{\min } \pi^{2}}=\frac{1}{q_{\max }+\varrho},
$$

then the needed inequality holds if

$$
2 K-\frac{1}{q_{\max }+\varrho} K^{2}-\kappa-q_{\max }-\frac{1}{2} \gamma^{-2} \geq 0,
$$

that always has a solution for

$$
\kappa+\frac{1}{2} \gamma^{-2} \leq \varrho
$$


In particular, for $\kappa+\frac{1}{2} \gamma^{-2}=\varrho$ we obtain:

$$
K=q_{\max }+\varrho=\frac{a_{\min } \pi^{2}}{\left(\Delta x^{m}\right)^{2}} .
$$

The inequality

$$
\dot{V}(t)+2 \kappa V(t)-\gamma^{2} \int_{0}^{\ell} R(x, t)^{2} d x \leq 0
$$

implies boundedness of the solutions $w(x, t)$ as in the proof of Proposition 4. We have proved the following theorem.

Theorem 11 Let assumptions 1 and 2 be satisfied. Let there exist $\kappa>0, K>0, \gamma>0$ and $\Delta x^{m}<\pi \sqrt{\frac{a_{\min }}{q_{\max }}}$ that satisfy the $L M I$

$$
\Phi \leq 0
$$

Then for the solutions of the closed-loop system (21), the interval inclusion (17) and the estimates on $\| z(\cdot, t)-$ $\underline{z}(\cdot, t)\left\|_{L^{2}(I, \mathbb{R})},\right\| \bar{z}(\cdot, t)-z(\cdot, t) \|_{L^{2}(I, \mathbb{R})}$ from Theorem 8 are valid and

$$
\begin{gathered}
\frac{1}{2} \int_{0}^{\ell} z^{2}(x, t) d x \leq e^{-2 \kappa\left(t-t_{0}\right)} \int_{0}^{\ell} w_{0}^{2}(x) d x+\frac{\gamma^{2}}{2 \kappa} \int_{0}^{\ell} R(x, t)^{2} d x \\
+\frac{\ell}{2}\left(\alpha^{2}(t)+\beta^{2}(t)\right) \quad \forall(x, t) \in I \times \mathcal{T}
\end{gathered}
$$

Remark 12 Note that qualitatively the above $L^{2}$ boundedness estimate for $z$ can also be obtained using static output feedback, however it can be rather conservative, and using the on-line calculated upper and lower observer bounds $\underline{z}$ and $\bar{z}$ we can deduce a tighter interval estimate on the state. This can be an important advantage for applications dedicated to state constrained problems (e.g. in reactors).

\section{Example}

In this section we will consider two applications of the proposed interval observer in order to compare the obtained results with the interval observer from [29] and the control from [12].

\subsection{Controller based on the interval observer}

Consider an academic example of (3) for

$$
\begin{gathered}
a(x)=\frac{1}{4}\left(1+\frac{3}{4} \sin (2 \pi x)\right), q(x)=5+\frac{1}{2} \cos (\pi x), \\
r(x, t)=\sin (\pi x)[\cos (2 t)+\epsilon(t)],|\epsilon(t)| \leq 1
\end{gathered}
$$

with $T=2$ and $\ell=1$, then $\epsilon$ is an uncertain part of the input $r$ (for simulation $\epsilon(t)=\cos (10 t)$ ), and

$\underline{r}(x, t)=\sin (\pi x)[\cos (2 t)-1], \bar{r}(x, t)=\sin (\pi x)[\cos (2 t)+1]$.

The uncertainty of initial conditions is given by the interval

$$
\underline{z}_{0}(x)=z_{0}(x)-1, \bar{z}_{0}(x)=z_{0}(x)+1,
$$

where $z_{0}(x)=5 \sin (\pi x)$, and for boundary initial conditions

$$
\begin{aligned}
& \underline{\alpha}(t)=\sin (2 t)-1, \bar{\alpha}(t)=\sin (2 t)+1, \\
& \underline{\beta}(t)=\sin (5 t)-1, \bar{\beta}(t)=\sin (5 t)+1,
\end{aligned}
$$

where $\alpha(t)=\sin (2 t)$ and $\beta(t)=\sin (5 t)$. Let $p=3$ with $x_{1}^{m}=0.3, x_{2}^{m}=0.6, x_{3}^{m}=0.8$, and

$$
\nu(t)=0.1[\sin (20 t) \sin (15 t) \cos (25 t)]^{\top},
$$

then $\nu_{0}=0.173$. In this case $a_{\min }=\frac{1}{16}, q_{\max }=5 \frac{1}{2}$. With these parameters, $q_{\max }$ is larger than $a_{\min } \frac{\pi^{2}}{\ell^{2}}$, which means that the system is unstable (the conditions of Proposition 4 fail to satisfy). The maximum distance between sensors is $\Delta x^{m}=0.3$, and the restriction (18) for the interval observer is still verified. Therefore, Theorem 8 can be used to construct an observer for the unstable system (3). Then, to stabilize it, following the conditions of Theorem 11, the control gain $K=3.2865$ was calculated, and the controls $u_{j}(t)$ on each interval $I_{i}=\left[x_{i}^{m}, x_{i+1}^{m}\right], i=\overline{0, p}$ with $x_{0}^{m}=0$ and $x_{p+1}^{m}=\ell$ were computed by (20).

For calculation of scalar product in space and for simulation of the discretized PDE in time, the implicit Euler method has been used with the step size $d t=0.01$. The results of a simultaneous interval estimation and control are shown in Fig. 1, where the red surface corresponds to $z(x, t)$, while green and blue ones represent $z(x, t)$ and $\bar{z}(x, t)$, respectively (20 and 100 points are used for plotting in space and in time).

In order to compare the proposed interval observer based control (20) with a static output feedback control

$$
u_{j}(t)=-K^{*} y_{j}(t)
$$

the feedback gain $K^{*}=4.8832$ is calculated following the result of the work [12]. Since the system (3) contains uncertainties in disturbances $r(x, t), \nu(t)$ and boundary conditions $\alpha(t), \beta(t)$, the static output feedback can guarantee only input-to-state stability in the sense of Proposition 4 with respect to the input $\tilde{r}(x, t)$, which contains all this incertitude. To compare the precision ensured by both controllers in our example, first, the $L^{2}$ 


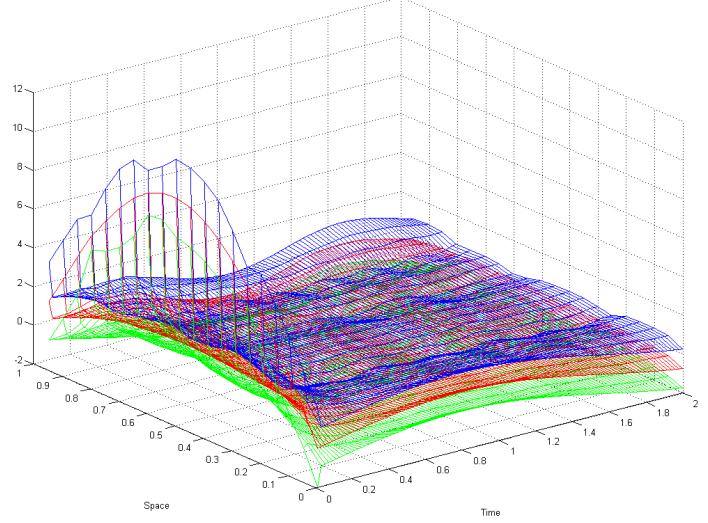

Fig. 1. The results of the interval observer based control of the heat equation for $N=20$ : the lower bound $\underline{z}(x, t)$, the state $z(x, t)$ and the upper bound $\bar{z}(x, t)$.

upper estimate of $z(x, t)$ for this feedback control is calculated as follows. Note that

$$
V(t)=\|z\|_{L^{2}(I, \mathbb{R})} \leq \ell \bar{z}^{2}(t),
$$

where

$$
\bar{z}(t)=\max _{x \in I}|z(x, t)|
$$

Clearly,

$$
z(t, x) \in[-\bar{z}(t), \bar{z}(t)] \quad \forall(x, t) \in I \times \mathcal{T} .
$$

From another side, the obtained $L^{2}$ estimates can be presented as

$$
V(t) \leq e^{-2 \delta\left(t-t_{0}\right)} V\left(t_{0}\right)+\gamma \int_{0}^{\ell}|\tilde{r}(x, t)|^{2} d x=\bar{V}(t),
$$

where $\bar{V}(t)$ can be calculated on-line for the given gain $K^{*}$ (it determines the values of parameters $\delta>0$ and $\gamma>0$ ) and the imposed upper bounds on $\tilde{r}(x, t)$. Second, for illustration we assume that $\bar{V}(t)=\ell \bar{z}^{2}(t)$, then the obtained bounds $[-\bar{z}(t), \bar{z}(t)]=$ $\left[-\sqrt{\ell^{-1} \bar{V}(t)}, \sqrt{\ell^{-1} \bar{V}(t)}\right]$ are shown in the Fig. 2 (black solid lines) together with the interval estimates of the proposed observer (14) (green and blue ones) for different instances of time. Red curves in the Fig. 2 represent the simulation of the stabilized heat equation (3) state using the interval observer, while the black dashed curves represent the state of (3) stabilized by output feedback (24). As we can conclude from this evaluation, the guaranteed bounds given by the interval observer based control are almost always more accurate than provided by the static feedback from $L^{2}$ estimates.

Remark 13 Note that since for calculation of solutions the finite-element discretization/approximation methods

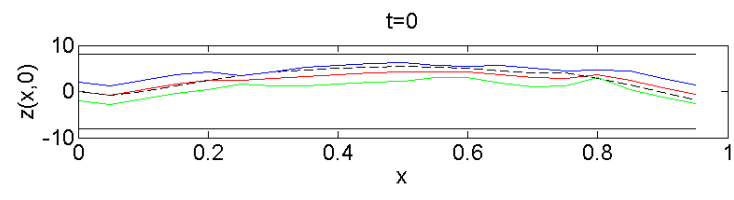

$t=0.5$

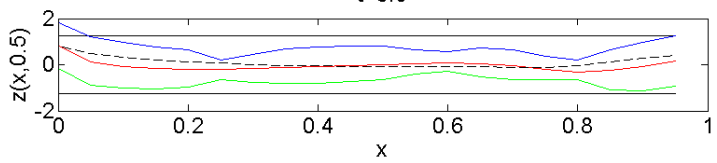

$t=1$

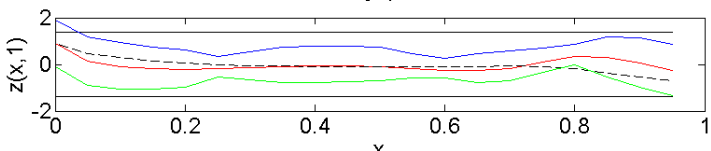

$\mathrm{t}=\mathrm{t}_{\mathrm{k}}$

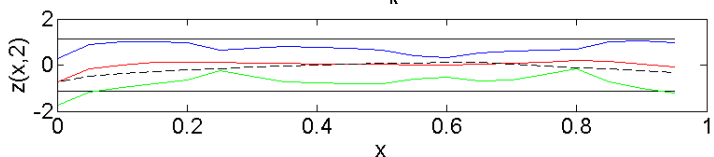

Fig. 2. The results of the interval observer based control and the $L^{2}$ estimate of the static output feedback control for the heat equation for different instants of time: $t=0,0.5,1,2$ for $N=20$. Here $z(x, \cdot)$ and $\bar{z}(x, \cdot)$ represent the interval observer bounds, $z(x, \cdot)$ is the stabilized state using observer, black dashed lines represent a state of (3) stabilized by output feedback $(24)$, black solid lines are $[-\bar{z}(t), \bar{z}(t)]$.

are used, then their error of approximation has to be taken into account in the final estimates in order to ensure the desired interval inclusion property for all $x \in I$ and $t \in \mathcal{T}$, see [29] where the result from [30] was applied for an evaluation of this error.

Remark 14 As mentioned in [12], there are no advantages of the Luenberger observer-based controller in the case of collocated sensors and actuators over the corresponding static output-feedback. However, as it is shown in this example, interval observer allows to achieve essentially lower state bounds than the corresponding static output-feedback.

\subsection{The interval observer comparison}

Consider a heat equation (3) with:

$$
\begin{gathered}
a(x)=2+0.7 \sin (\pi x), q(x)=0.5 \sin (0.5 x), \\
r(x, t)=r_{1}(x) r_{2}(t), r_{1}(x)=2 \cos (3 \pi x),\left|r_{2}(t)\right| \leq 1,
\end{gathered}
$$

$T=10$ and $\ell=1$. Here $r_{2}$ is an uncertain part of the input $r$ (for simulation $r_{2}(t)=\cos (15 t)$ ), and

$$
\underline{r}(x, t)=-\left|r_{1}(x)\right|, \bar{r}(x, t)=\left|r_{1}(x)\right| .
$$


The uncertainty of initial conditions is given by the interval

$$
\underline{z}_{0}(x)=z_{0}(x)-1, \bar{z}_{0}(x)=z_{0}(x)+1,
$$

where $z_{0}(x)=\cos (5 \pi x)$, and the boundary conditions $\alpha(t)$ and $\beta(t)$ are assumed to be 0 , since the approach from [29] does not employ nonzero conditions. Let $p=3$ with $x_{1}^{m}=0.3, x_{2}^{m}=0.5, x_{3}^{m}=0.8$, and

$$
\nu(t)=0.2[\sin (20 t) \sin (15 t) \cos (25 t)]^{\top},
$$

then $\nu_{0}=0.2$. In this case $\Delta x^{m}=0.3, a_{\min }=1.3$ $q_{\max }=0.5$ and the restriction (18) is satisfied. Take $\Delta=$ $\{0, h, 2 h, \ldots, 1-h, 1\}$ with $h=1 / N^{\prime}$, and a pyramidal basis

$$
\Phi_{i}(x)= \begin{cases}0 & x \leq x_{i-1}, \\ \frac{x-x_{i-1}}{x_{i}-x_{i-1}} & x_{i-1}<x \leq x_{i}, \\ \frac{x_{i+1}-x}{x_{i+1}-x_{i}} & x_{i}<x \leq x_{i+1}, \\ 0 & x \geq x_{i+1}\end{cases}
$$

for $i=0, \ldots, N=N^{\prime}$ (it is assumed $x_{-1}=-h$ and $\left.x_{N+1}=1+h\right)$. For simulation we took $N=20$, then the approximated dynamics from [29] is an observable system, and assume that the error of approximation for both approaches $\varrho h^{s+1}\left(l_{1}+l_{2}\right)=0.1$. For the Galerkin approximation approach [29] the matrix $\mathcal{L}$ has been chosen to ensure distinct eigenvalues of the matrix $A-\mathcal{L} C$ in the interval $[-30.9,-0.67]$, then $S^{-1}$ has been composed by eigenvectors of the matrix $A-\mathcal{L} C$ and the matrix $D$ has been selected diagonal (all these matrices are defined in [29]).

As before, for the calculation of scalar product in space and for simulation of the discretized PDE in time, the implicit Euler method has been used with the step size $d t=0.01$ for the PDE interval observer, and the explicit one with the same step for the approximation approach. The results of comparison of the two approaches, the present and the approximation one from [29], are shown in Fig. 3, where the red lines corresponds to $z(x, \cdot)$, while green and blue ones represent $\underline{z}(x, \cdot)$ and $\bar{z}(x, \cdot)$, respectively, at the instances $t=0,1,5,10$. From this figure one can clearly notice that the obtained interval for the state is more precise with the PDE interval observer approach (14).

\section{Conclusion}

Taking a heat equation with Dirichlet boundary conditions, a method of design of interval observers is proposed, which is not based on a finite-element approximation. The design employs the positivity of solutions of the heat equation proposed in [32]. The proposed interval observer is used for stabilization of an uncertain PDE system. The efficiency of the approach is demonstrated through numerical experiments.

\section{PDE interval observer}
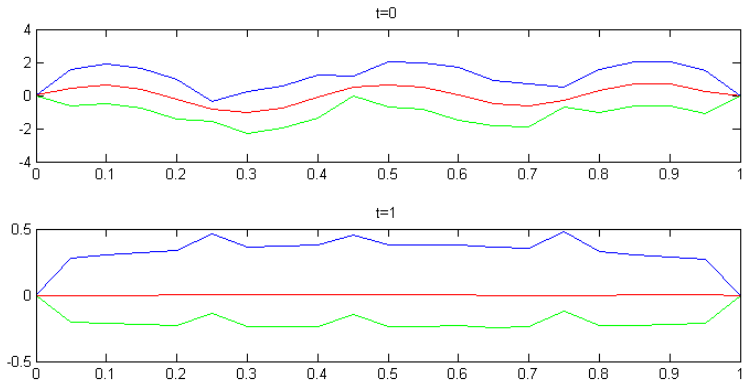

$t=5$

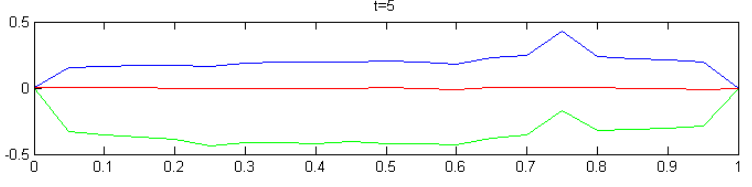

$\mathrm{t}=$

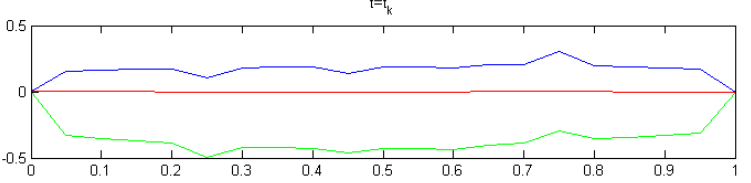

2. Approximation approach for 10 design

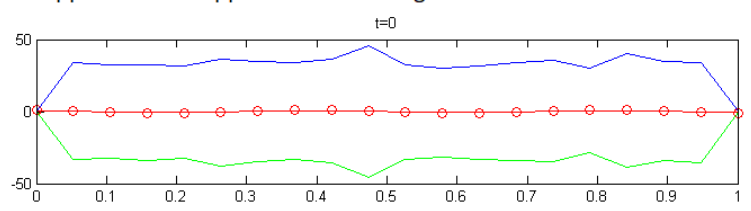

$t=1$
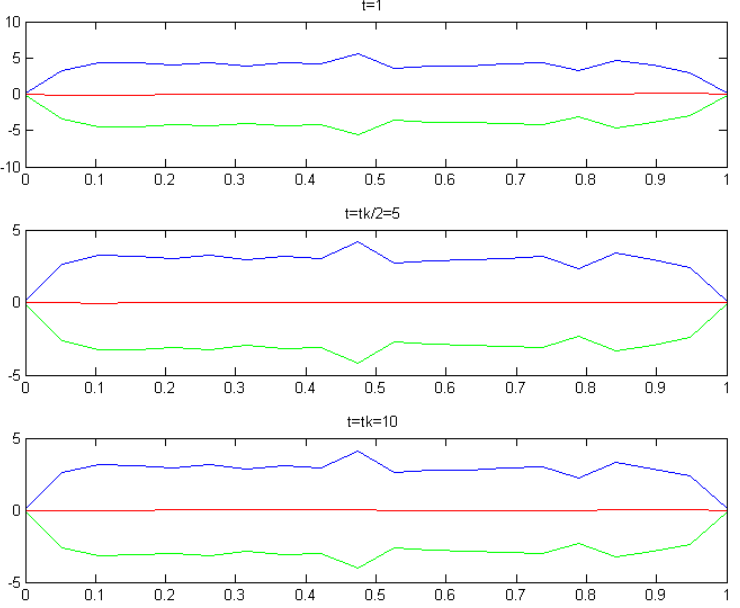

Fig. 3. The results of 1) the PDE interval observer (14) and 2 ) the approximation approach interval observer from [28], $N=20$. Here the lower bound is $\underline{z}(x, \cdot)$, the state is $z(x, \cdot)$ and the upper bound is $\bar{z}(x, \cdot)$.

For future developments, more complex uncertainty of PDE equation can also be incorporated in the design procedure and the approach can be extended to PDEs with Neumann, Robin, or mixed boundary conditions. Possibility of averaged measurements and the corresponding positivity conditions can also be considered as a direction of future research. 


\section{Acknowledgements}

This work is partially supported by the Government of Russian Federation (Grant 08-08), by the Ministry of Science and Higher Education of Russian Federation (passport of goszadanie no. 2019-0898) and by Israel Science Foundation 1128/14.

\section{References}

[1] T. Meurer, K. Graichen, and E.-D. Gilles, eds., Control and Observer Design for Nonlinear Finite and Infinite Dimensional Systems, vol. 322 of Lecture Notes in Control and Information Sciences. Springer, 2005.

[2] T. Fossen and H. Nijmeijer, New Directions in Nonlinear Observer Design. Springer, 1999.

[3] G. Besançon, ed., Nonlinear Observers and Applications, vol. 363 of Lecture Notes in Control and Information Sciences. Springer, 2007.

[4] K. Bredies, C. Clason, K. Kunisch, and G. von Winckel, Control and optimization with PDE constraints, vol. 164 of International Series of Numerical Mathematics. Basel: Birkhäuser, 2013.

[5] A. Smyshlyaev and M. Krstic, Adaptive Control of Parabolic PDEs. Princeton University Press, 2010.

[6] J. Alvarez and G. Stephanopoulos, "An estimator for a class of non-linear distributed systems," International Journal of Control, vol. 5, no. 36, pp. 787 802, 1982.

[7] D. Dochain, "State observers for tubular reactors with unknown kinetics," Journal of Process Control, vol. 10, pp. 259-268, 2000.

[8] A. Vande Wouver and M. Zeitz, Encyclopedia of Life Support Systems (EOLSS), ch. State estimation in distributed parameter systems. Eolss Publishers, 2002. Developed under the Auspices of the UNESCO.

[9] G. Hagen and I. Mezic, "Spillover stabilization in finite-dimensional control and observer design for dissipative evolution equations," SIAM Journal on Control and Optimization, vol. 2, no. 42, pp. 746768, 2003.

[10] Z. Hidayat, R. Babuska, B. D. Schutter, and A. Nunez, "Observers for linear distributedparameter systems: A survey," in Proc. IEEE Int. Symp. Robotic and Sensors Environments (ROSE), pp. 166-171, 2011.

[11] T. Ahmed-Ali, F. Giri, M. Krstic, and F. LamnabhiLagarrigue, "Observer design for a class of nonlinear ODE-PDE cascade systems," Systems \& Control Letters, vol. 83, pp. 19-27, 2015.

[12] E. Fridman and A. Blighovsky, "Robust sampleddata control of a class of semilinear parabolic systems," Automatica, vol. 48, pp. 826-836, 2012.

[13] A. Schaum, J. A. Moreno, E. Fridman, and J. Alvarez, "Matrix inequality-based observer design for a class of distributed transport-reaction systems,"
International Journal of Robust and Nonlinear Control, vol. 24, no. 16, pp. 2213-2230, 2014.

[14] E. Fridman, "Observers and initial state recovering for a class of hyperbolic systems via Lyapunov method," Automatica, vol. 49, pp. 2250-2260, 2013.

[15] A. Selivanov and E. Fridman, "Delayed point control of a reaction-diffusion PDE under discretetime point measurements,," Automatica, vol. 96, pp. 224-233, 2018.

[16] E. Fridman and N. Bar Am, "Sampled-data distributed $H^{\infty}$ control of transport reaction systems," SIAM Journal on Control and Optimization, vol. 51, no. 2, pp. 1500-1527, 2013.

[17] A. Pisano and Y. Orlov, "On the ISS properties of a class of parabolic DPS with discontinuous control using sampled-in-space sensing and actuation," Automatica, vol. 81, pp. 447-454, 2017.

[18] J.-W. Wang, Y.-Q. Liu, and C.-Y. Sun, "Pointwise exponential stabilization of a linear parabolic PDE system using non-collocated pointwise observation," Automatica, vol. 93, pp. 197-210, 2018.

[19] L. Jaulin, "Nonlinear bounded-error state estimation of continuous time systems," Automatica, vol. 38, no. 2, pp. 1079-1082, 2002.

[20] M. Kieffer and E. Walter, "Guaranteed nonlinear state estimator for cooperative systems," Numerical Algorithms, vol. 37, pp. 187-198, 2004.

[21] B. Olivier and J. Gouzé, "Closed loop observers bundle for uncertain biotechnological models," Journal of Process Control, vol. 14, no. 7, pp. 765$774,2004$.

[22] M. Moisan, O. Bernard, and J. Gouzé, "Near optimal interval observers bundle for uncertain bioreactors," Automatica, vol. 45, no. 1, pp. 291-295, 2009.

[23] T. Raïssi, G. Videau, and A. Zolghadri, "Interval observers design for consistency checks of nonlinear continuous-time systems," Automatica, vol. 46, no. 3, pp. 518-527, 2010.

[24] T. Raïssi, D. Efimov, and A. Zolghadri, "Interval state estimation for a class of nonlinear systems," IEEE Trans. Automatic Control, vol. 57, no. 1, pp. 260-265, 2012.

[25] D. Efimov, L. Fridman, T. Raïssi, A. Zolghadri, and R. Seydou, "Interval estimation for LPV systems applying high order sliding mode techniques," $A u$ tomatica, vol. 48, pp. 2365-2371, 2012.

[26] J. Gouzé, A. Rapaport, and M. Hadj-Sadok, "Interval observers for uncertain biological systems," Ecological Modelling, vol. 133, pp. 46-56, 2000.

[27] H. Perez and S. Moura, "Sensitivity-based interval PDE observer for battery SOC estimation," in Proc American Control Conference (ACC), pp. 323-328, 2015.

[28] T. Kharkovskaya, D. Efimov, A. Polyakov, and J.P. Richard, "Interval observers for PDEs: approximation approach," in Proc. 10th IFAC Symposium on Nonlinear Control Systems (NOLCOS), (Monterey), 2016. 
[29] T. Kharkovskaya, D. Efimov, A. Polyakov, and J.-P. Richard, "Design of interval observers and controls for PDEs using finite-element approximations," $A u$ tomatica, vol. 93, pp. 302-310, July 2018.

[30] M. Wheeler, " $l \infty$ estimates of optimal orders for Galerkin methods for one-dimensional second order parabolic and hyperbolic equations," SIAM J. Numer. Anal., vol. 10, no. 5, pp. 908-913, 1973.

[31] A. Rauh, J. Kersten, and H. Aschemann, "An interval observer approach for the online temperature estimation in solid oxide fuel cell stacks," in Proc. European Control Conference (ECC), (Limassol), 2018.

[32] H.-M. Nguyen and J.-M. Coron, "Null controllability and finite time stabilization for the heat equations with variable coefficients in space in one dimension via backstepping approach," INFOSCIENCE, 2016. EPFL-ARTICLE-214990.

[33] T. Kharkovskaya, D. Efimov, E. Fridman, A. Polyakov, and J.-P. Richard, "On design of interval observers for parabolic PDEs," in Proc. 20th IFAC World Congress, (Toulouse), July 2017.

[34] G. Hardy, J. Littlewood, and G. Polya, Inequalities. Cambridge University Press, 1988.

[35] N. Bar Am and E. Fridman, "Network-based $H_{\infty}$ filtering of parabolic systems," Automatica, vol. 50, no. 12, pp. 3139-3146, 2014.

[36] A. Pazy, Semigroups of linear operators and applications to partial differential equations. Springer, 1983.

[37] S. N. Dashkovskiy, D. V. Efimov, and E. D. Sontag, "Input-to-state stability and allied system properties," Automation and Remote Control, vol. 72, no. 8, p. 1579, 2011.

[38] S. Dashkovskiy and A. Mironchenko, "Input-tostate stability of infinite-dimensional control systems," Mathematics of Control, Signals, and Systems, vol. 25, no. 1, pp. 1-35, 2013.

[39] V. Thomée, Galerkin Finite Element Methods for Parabolic Problems. Berlin: Springer, 2006.

[40] A. Friedman, Partial differential equations of parabolic type. Englewood Cliffs, N.J.: PrenticeHall, Inc., 1964.

[41] R. Curtain and H. Zwart, An introduction to infinite-dimensional linear systems theory. New York: Springer-Verlag, 1995.

[42] D. Efimov, T. Raïssi, and A. Zolghadri, "Control of nonlinear and LPV systems: interval observerbased framework," IEEE Trans. Automatic Control, vol. 58, no. 3, pp. 773-782, 2013. 